\title{
Diseño de ciclovías para ciudades intermedias, una propuesta para Loja
}

\section{Design of ciclovias for intermediate cities, a proposal for Loja}

\author{
Verónica Alexandra Muñoz Sotomayor \\ David Betancourt, \\ Wilson Jaramillo Sangurima \\ Universidad Internacional del Ecuador, Ecuador
}

Autor para correspondencia: valex.munoz@gmail.com

Fecha de recepción: 06 de Septiembre de 2016 - Fecha de aceptación: 10 de Diciembre de 2016

\section{Resumen}

La sostenibilidad es un concepto que ha tomado mayor protagonismo en las últimas décadas en diferentes ámbitos de desarrollo; en la dimensión urbana, la movilidad merece cada día mayor atención, ya que de ahí deriva el problema central. La presente investigación, aborda la problemática de movilidad, del modelo urbano característico de algunas ciudades intermedias de Latinoamérica, donde las calles del casco central soportan la presión causada por incremento del parque automotor generándose congestión vehicular. El presente estudio, propone mejorar la movilidad urbana de la ciudad de Loja - Ecuador, implementando e impulsando el uso de ciclovías como parte de una política de movilidad sustentable, a través de una metodología de estudio que considera un enfoque de tipo cuantitativo, sobre el cual se aplica un diseño de investigación no experimental de los aspectos físicos y morfológicos de la ciudad , considerando cuatro instancias, cuyo escenario al ser simulado por un software de tránsito, arroja como resultado reducción en la congestión vehicular e impacto ambiental; que concluye en una propuesta de prioridad, tipologías y estrategias de diseño y servicios de ciclovía para el caso de estudio.

Palabras claves: ciclovía; desarrollo sustentable; movilidad sustentable

\begin{abstract}
Sustainability is a concept that has gained greater prominence in recent decades in various fields of development; in the urban dimension, the main problem derive form this matter. In the intermediate cities, the streets of the city endure the pressure caused by the increase of motor vehicles generating traffic congestion, pollution, consumption of time and economic expenditure to the Population; situation that tends to worsen every day if actions that encourage changes in mobility habits are not taken. The present research aims to improve urban mobility in Loja's city, implementing and promoting the use of bikeways as part of a policy of sustainable mobility, applying a quantitative approach, on which it is applied a design of experimental transactional investigation, considering four instances whose stage is being simulated by a transit software resulting on the reduction of traffic congestion and environmental impact. As result it was obteained congestional vehicular reduction and a reduce environmental impact, through a proposal of priority, tipology and desing strategies and bikeways services for this case of study.
\end{abstract}

Key words: ciclovía; bicycle; sustainable development; local development 


\section{Introducción}

Según Colmenares (2007), las acciones a emprender para conseguir soluciones en el marco de una movilidad sustentable, deben considerar tres factores: primero el social, ya que debe permitir la accesibilidad de toda la población, generando equidad social en los sistemas de transporte; segundo el ecológico, porque debe proteger al medio ambiente y consecuentemente a la salud de la población, reduciendo las emisiones de gases de efecto invernadero que genera el transporte motorizado; y tercero el económico, por tanto debe satisfacer la economía promoviendo el desarrollo y la competitividad, reduciendo y regulando las necesidades de varios sectores económicos.

El análisis de estos factores dentro de la movilidad urbana de la ciudad, motivan a promover la movilidad a pie, en bicicleta, en transporte público colectivo, a mejorar la ocupación y uso de los automóviles, y a considerar el empleo de la innovación tecnológica en vehículos y combustibles.

Por tales razones, uno de los temas que cada día gana más importancia en las ciudades del mundo, es el promover el uso de la bicicleta como uno de los principales modo de transporte, considerando que potencian algunos beneficios para la población como la salud, ya que puede mejorar la salud física y emocional; ayuda al funcionamiento del sistema cardiovascular, tonifica los músculos y mejora la capacidad pulmonar; reduce los niveles de colesterol en la sangre; ayuda a mejorar la coordinación motriz; es un excelente ejercicio aeróbico que combate los riesgos de sufrir sobrepeso y obesidad; reduce los niveles de estrés y mejora el estado de ánimo. Desde el punto de vista ecológico, el uso de la bicicleta ayuda a reducir los niveles de contaminación ambiental y sus niveles de monóxido y dióxido de carbono, hidrocarburos y otras partículas que favorecen la contaminación del aire.

Un denominador común suele presentarse en las ciudades intermedias; clasificadas en esta categoría más que por su peso demográfico, por su características cualitativas, una de ellas ese el grado significativo de centralidad. La ciudad de Loja, fundada en el año de 1548 e independizada en 1820, se encuentra ubicada al sureste del Ecuador y forma parte de la denominada región sur o zona 7. Urbanísticamente se caracteriza por estar constituida por un trazado ortogonal (trazado damero), el cual se origina en la plaza central seguido con el trazado de manzanas rectangulares y cuadradas, con sus calles principales originadas por la topografía (pendientes mínimas) y su hidrografía (ríos Malacatos y Zamora) cuyo caudal fluye de sur a norte; y se extiende hacia el territorio montañoso que delimita el valle donde se desenvuelve el centro de la ciudad. Tiene una población según el censo INEC (2010) de 170280 habitantes y es capital de provincia, con lo cual es considerada una de las ciudades intermedias del Ecuador.

La presencia de un número considerable de comercios, centros educativos, entidades públicas y privadas, provoca el deseo de viajes de los habitantes hacia el centro histórico de la ciudad, lo cual combinado con el crecimiento de la población y el parque automotor (Programa de Naciones Unidas para el Medio Ambiente, Municipalidad de Loja y Naturaleza y Cultura Internacional, 2007), genera la ocurrencia de una gran presión sobre la capacidad de calles y avenidas, lo que se traduce en problemas de congestión vehicular, contaminación ambiental y consumo de tiempo en la vida de la población, situación que se prevé se seguirá agravando, si no 
se emprende en acciones que motiven el cambio en los hábitos de movilidad, en procura de un desarrollo sustentable.

Entre los principales problemas que presenta la ciudad de Loja para el uso de la bicicleta está la falta de espacio físico adecuado y seguro, ya que únicamente se puede evidenciar la existencia de senderos que pueden ser utilizados por ciclistas en los parques lineales de la ciudad, no así en la vía pública; por lo tanto la actividad ciclística es aceptada culturalmente con una actividad recreativa y no como una alternativa de movilidad urbana; entonces, ¿cómo incorporar una movilidad sostenible que incluya el uso de la bicicleta en una estructura vial antigua y morfológicamente limitada?

En base a lo expuesto se evidencia la necesidad de promover y evaluar las condiciones adecuadas, que incentiven el uso de la bicicleta como uno de los principales modos de transporte utilizados por los habitantes de la ciudad de Loja, emprendiendo en un trabajo que responda a métodos científicos que permitan resolver este problema, lo que lleva a plantear la siguiente pregunta de investigación, ¿Se puede mejorar la movilidad urbana de la ciudad de Loja (como modelo de ciudad intermedia), implementando e impulsando el uso de ciclovías como parte de una política de movilidad sustentable?; pregunta que pretende responderse bajo la hipótesis de investigación que la implementación de una ciclovía en la ciudad de Loja, ayuda a reducir la congestión vehicular y el impacto ambiental, siendo considerada como una de las mejores opciones de movilidad sustentable.

\section{Discusión Teórica}

Actualmente las ciudades se han convertido en verdaderos consumidores de recursos naturales, el impacto que generan a los ecosistemas son globales afectando a todo ser vivo del planeta, y todo parece indicar que seguirá agravándose si no se aplican políticas de sustentabilidad y sostenibilidad que ayuden a detener este impacto global.

La sustentabilidad ha crecido a lo largo de estos últimos años, abarcando desde aspectos micros a macros en cuanto al desarrollo de las ciudades. En escala de ciudad, se liga estrechamente a la ecología urbana ya que la misma beneficia a que se incorpore la naturaleza a la urbe por ende en la ciudad (Romero, Toledo, \& Vasquez , 2001), es así que la ecología urbana trata las ciudades como "ecosistemas, es decir, como sistemas integrados con relaciones multivariadas entre los componentes internos y con el contexto" (Rueda, 1995).

Los sistemas de movilidad han sido desde la concepción del urbanismo moderno, el talón de Aquiles del desarrollo local por el mismo hecho que "el crecimiento del número de vehículos motorizados está en la raíz misma de la movilidad" (Gakenheimer, 1998), de ahí la complejidad de predecir o controlar los efectos negativos que el parque automotor pueda ejercer sobre el medio ambiente urbano. Esta cuestión se aborda a partir de las políticas de la sustentabilidad del siglo XIX, bajo la disciplina de la movilidad sustentable, cuyas características pueden ser establecidas en dos ejes, el uno que corresponde a los lugares por donde circulan los diferentes tipos de transporte, y el otro por los tipos de transporte que existen

\section{La movilidad como base de la ciudad sustentable}


La sustentabilidad en la ciudad no solo se refiere a la materialidad, sino a la educación de los habitantes con respecto a ello, por tal razón, se necesitan más políticas urbanas sustentables que incluyan, involucren e inviten a la ciudadanía a formar parte de este cambio para el bien común. Esta característica define a la ciudad sostenible, ya que propone la integración de los aspectos sociales, ambientales y económicos, ésta integración desarrolla, fomenta y difunde proyectos y programas para la ciudad, para el uso y el buen vivir de los habitantes, satisfaciendo sus expectativas y deseos (Naredo, 1997).

Dado que la movilidad es el principal conflicto de la ciudad actual, es precisamente el punto de partida para la sustentabilidad urbana, que se va plasmando en una serie de estrategias que ayudan a consolidar la ciudad a través de cada uno de sus sistemas, los cuales favorecen a la organización de la misma para el bienestar de las personas; dichas estrategias deben proteger y multiplicar los recursos, cuidar la salud humana y el ambiente, mejorar los medios de transporte y a través de estas políticas sostener la economía local (Hunt, 2005).

Por lo tanto, se complementa la movilidad sustentable con la movilidad ecológica mediante sistemas de transporte que dejen un mínimo de contaminación ambiental y de afectaciones a la salud, como es el automóvil eléctrico y principalmente la bicicleta como transporte no motorizado (Acosta, 2004).

\section{La movilidad sustentable}

Las características de movilidad sustentable pueden ser establecidas en dos ejes, el uno que corresponde a los lugares por donde circulan los diferentes tipos de transporte, y el otro por los tipos de transporte que existen. Dentro del primer eje se establece que la jerarquía de movilización ubica en primero, segundo y tercer lugar al peatón, la bicicleta y el transporte público colectivo respectivamente; por lo tanto es evidente la prioridad que debe darse al lugar físico de circulación de esta pirámide de movilidad. Las políticas y proyectos de la movilidad sustentable deben ser integrales, considerando que, una estrategia de fortalecimiento de un modo de transporte debe ser planificado con la coexistencia simultánea de otros.

En cuanto concierne al diseño de ciclovías, se parte de la compresión de su definición, entendiendo que se trata de una red vial de infraestructura pública exclusiva para la circulación de bicicletas. Con esta finalidad se retoma la bicicleta como transporte sustentable no motorizado, y debe cumplir con los dispositivos de seguridad en cuanto a la circulación y operación de bicicletas en dichas ciclovías, con el propósito de proteger al ciclista, al peatón que está junto a la infraestructura y proteger el medio ambiente, aplicando criterios técnicos (Plan Nacional de Ciclovias del Ecuador, Ministerio de Transporte y Obras Publicas, 2012).

De acuerdo a las características de las ciclovías, describimos la siguiente clasificación:

- Ciclovías reservadas: Son infraestructuras planificadas y creadas para bicicletas y uso de peatones, generalmente se encuentran ubicadas en zonas urbanas y en grandes parques 
- Ciclovías segregadas: Son vías ciclísticas que están integradas en donde existió anteriormente un carril vehicular, en estas no se permiten peatones ya que están destinadas exclusivamente para ciclistas; por el tipo de ubicación se las conoce como carril bicicleta (en la calle), acera bicicleta (en aceras), y ciclovía en espaldón.

- Ciclovías compartidas: Son vías ciclistas que comparten el carril con el vehículo, tienen que estar dotadas de una adecuada señalización, el ciclista tiene que ir en el centro del carril, y la velocidad del vehículo no tiene que superar las $30 \mathrm{~km} / \mathrm{h}$ máximo

- Senderos para bicicletas: son caminos para ciclistas no necesariamente asfaltados, y comúnmente se las encuentra en parques nacionales con extensos recorridos.

- Ciclovías recreativas: Son calles vehiculares cerradas durante horas o días para abrirlas a los peatones, ciclistas y deportistas (Americas, 2012).

El diseño de ciclovías, para cualquiera de las tipologías, se rige a normativas técnicas, de medidas mínimas y máximas, señalización vertical y horizontal, límites de velocidad y diseño formal y funcional en general (Plan Nacional de Ciclovias del Ecuador, Ministerio de Transporte y Obras Publicas. 2012); en realidad muchas de los diseños de ciclovías en las ciudades se reducen a una propuesta informal, justificado en la oferta netamente recreativa, sin otorgarle la categoría de movilidad urbana cotidiana.

\section{Métodos}

Para realizar la investigación se estableció como ambiente de trabajo el Municipio de Loja a través de la Unidad Municipal de Transporte Terrestre, Tránsito y Seguridad Vial "UMTTTSV", la cual tiene como tarea planificar, regular y controlar el transporte terrestre, tránsito y seguridad vial en el cantón Loja. Con el propósito de responder a la pregunta de investigación y cumplir con el objetivo del estudio, se empleó un enfoque de investigación de tipo cuantitativo considerando un diseño de investigación no experimental de tipo transeccional.

En la primera etapa de la investigación se realizó un diagnóstico general de la situación actual de la movilidad en la ciudad de Loja, identificando las características geométricas y jerárquicas de la red vial, intersecciones y cruces conflictivos, congestión vehicular, déficit de estacionamientos, medios de transporte, red de transporte urbano y contaminación ambiental. Así mismo se investigó sobre proyectos futuros a ser implementados por el Municipio de Loja, en el cual se contemple la implementación de ciclovías, como en el caso específico del proyecto de Regeneración Urbana que actualmente se está ejecutando.

Actualmente, en la ciudad existen dos proyectos en planificación por parte del Ilustre Municipio de Loja:

- Ciclovía por parte del Plan de Regeneración Urbana (centro de la Ciudad).

- Ciclovía por parte de la Unidad Municipal de Tránsito y Transporte Terrestre (Orillas del río Malacatos y del río Zamora).

La presente investigación, incluye estos dos proyectos en el análisis y simulación del funcionamiento de movilidad para la ciudad de Loja, y evalúa la suficiencia de la oferta de este sistema. 


\section{Determinación de instancias de intervención}

Con la información recabada y debidamente estudiada, y considerando especialmente las características del relieve de la ciudad, se sectorizó y definió en cuatro a las instancias del proceso de planificación, lo que permitió estudiar, generar y simular una propuesta de implementación de ciclovías de manera integral para la ciudad de Loja.

La clasificación del suelo urbano en instancias es el producto de una ponderación de variables que caracterizan la morfología de la ciudad de Loja: densidad poblacional, topografía, equipamientos urbanos, flujo vehicular y peatonal, fueron factores valorados para delimitar las instancias (ilustración 1); las mismas que no solo determinan un orden jerárquico de demanda sino también las características de la vialidad ciclística.

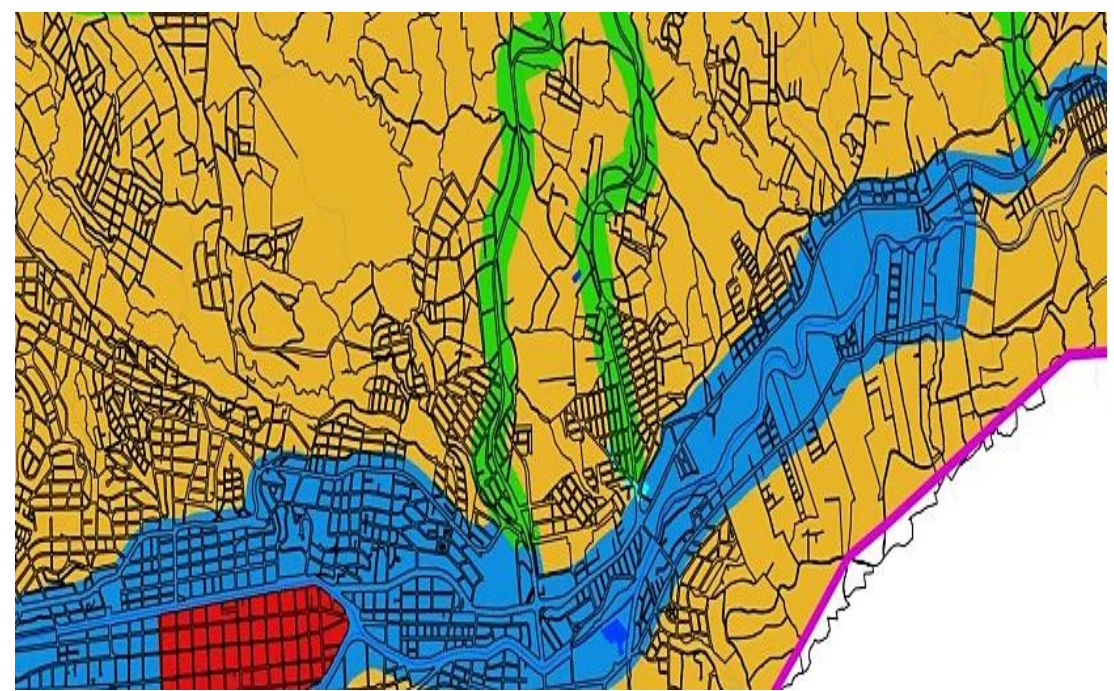

Ilustración 1: Generación de instancias de análisis e intervención de acuerdo a las características morfológicas y poblacionales de la ciudad de Loja. Fuente: El autor

La primera instancia contempla el estudio de las calles céntricas de la ciudad o centro histórico, la segunda instancia considera el estudio de calles y avenidas que presentan relieves mínimos en la ciudad y que se unen con las calles céntricas. La tercera instancia estudia las calles locales y colectoras no consideradas en la instancia anterior que permitan integrar al $85 \%$ de los barrios de la ciudad, y la cuarta instancia analiza las vías expresas y arteriales con la finalidad de completar el circuito de ciclovías que abarque toda la ciudad.

Una vez definidas las instancias se procedió a realizar un análisis de tráfico vehicular en las calles contempladas en las instancias 1 y 2, para lo cual, se realizó conteos de flujo vehicular y peatonal respetando metodologías propias de la ingeniería de tránsito, posteriormente se procedió a tabular la información recogida; se ingresó los datos a un software de simulación de tránsito (Synchro) y se procedió a analizar los resultados considerando el escenario de implementación de una ciclovía.

\section{Evaluación de aceptación de la población}


Como se había mencionado anteriormente, la aceptación social de este tipo de proyectos es fundamental para el funcionamiento eficiente y planificado; por ello conocer el interés de uso de este servicio es menester para conocer si el proyecto de movilidad sustentable tendrá una correspondencia con una cultura sustentable que poco a poco se vaya insertando en la sociedad.

La evaluación de aceptación se llevó a cabo mediante la aplicación de una encuesta dirigida a los peatones y usuarios del espacio público (con una muestra de 100 personas), que permitan establecer las principales necesidades con respecto a la implementación de una infraestructura ciclística como alternativa de una movilidad sustentable como son: la frecuencia con la que la ciudadanía utiliza la bicicleta, tipos de transporte que utilizan con mayor frecuencia, tiempo en su desplazamiento, entre otros parámetros.

\section{Resultados y Discusión}

La movilidad actual en la ciudad de Loja se percibe en 3 características, aumento del parque automotor, deficiente control del uso del suelo, aumento de viajes al centro de la ciudad. En la actualidad la necesidad de un desplazamiento más saludable, rápido, eficiente y económico, se ha visto empañado por la facilidad que existe para adquirir un vehículo motorizado, lo que a su vez genera un crecimiento del parque automotor, aglomeración de los equipamientos y actividades en el centro de la ciudad, hecho que produce un elevado índice de congestionamiento vehicular e inseguridad peatonal al superar la capacidad de la infraestructura vial y peatonal.

La unidad municipal de tránsito y transporte terrestre de la ciudad de Loja en el año 2013, nos señala los principales problemas que tiene la ciudad:

- Excesiva fragmentación en el corredor central de transporte, mediante cruces y puentes vehiculares.

- Deficiente señalización en las avenidas

- El estacionamiento de vehículos particulares en el corredor exclusivo de transporte urbano, ocasiona congestionamiento debido a la presencia de locales comerciales a lo largo de las tres avenidas más importantes de la ciudad.

- Frecuentes accidentes vehiculares y atropellamientos en el corredor central.

- Los lugares de mayor congestión vehicular se encuentran desde la Av. Mercadillo hasta el puente de LEA con colas vehiculares que alcanzan aproximadamente los $120 \mathrm{~m}$ de longitud en horas pico, con velocidades de $15 \mathrm{~km}$ por hora.

Contrastando los resultados del análisis planteado por esta institución con las evaluaciones realizadas con la simulación de software se pudieron generar los siguientes resultados:

La movilidad y el transporte en la ciudad de Loja representan el principal problema urbano, el cual se origina con mayor énfasis en el centro histórico y se expande a sus alrededores específicamente en las instancias 1 y 2 de norte a sur, debido a su topografía y vías principales. Existen dos proyectos de ciclovía que actualmente se están planificando en el Ilustre Municipio de Loja, que de acuerdo a la demanda y oferta planteada se pueden concluir lo siguiente: 
- Ciclovía Plan de Regeneración Urbana: Esta ciclovía planificada en la primera instancia solo tiene como propuesta carriles ciclísticos físicos, no contempla un proyecto de carril compartido; por tal motivo para complementarla se deben implementar carriles compartidos en el centro de la ciudad para tener circuitos que se interconecten y potencien el uso de la bicicleta como medio de transporte urbano.

- Ciclovía Unidad Municipal de Tránsito y Transporte Terrestre: Esta ciclovía representa el circuito troncal de la ciudad de Loja que va de Norte a Sur y viceversa, pese a ello, aún se encuentra desvinculada del servicio requerido hacia el centro de la ciudad, por lo tanto es necesario complementar el proyecto con circuitos que conecten esta infraestructura planificada tanto hacia el centro como hacia los barrios ubicados al otro lado de ambos ríos.

En lo que respecta a la aceptación social, el $87 \%$ de la población encuestada cuya edad oscila entre los 17 y 37 años de edad, de los cuales el $48 \%$ son mujeres y el 52\% hombres, estaría dispuesta a utilizar infraestructura ciclística, si esta ofreciera espacios aptos con circuitos especiales, estacionamientos y seguridad. En la actualidad el $98 \%$ de esta población no utiliza la bicicleta para realizar sus actividades diarias, ya que el $72 \%$ manifiesta que cuando usan la bicicleta no son respetados por los conductores de vehículos particulares o de transporte público. El 23\% utilizan la bicicleta para realizar actividades de recreación en parques, senderos o fuera de la ciudad, el $81 \%$ utilizaría una ciclovía empleando una bicicleta propia o rentada como parte del programa de bicicleta pública, el $90 \%$ son conscientes que el uso de la bicicleta mejoraría su estado de salud y condición física, el 74\% le daría prioridad de uso si reduciría el tiempo de desplazamiento con respecto al vehículo y un $89 \%$ es consiente que al utilizar la bicicleta aportaría a reducir la contaminación ambiental.

En cuanto a las características del flujo vehicular existente en las calles de la ciudad de Loja, se pudo identificar las intersecciones que presentan niveles de congestión vehicular alto, medio y bajo, como se puede observar en la ilustración 2 .

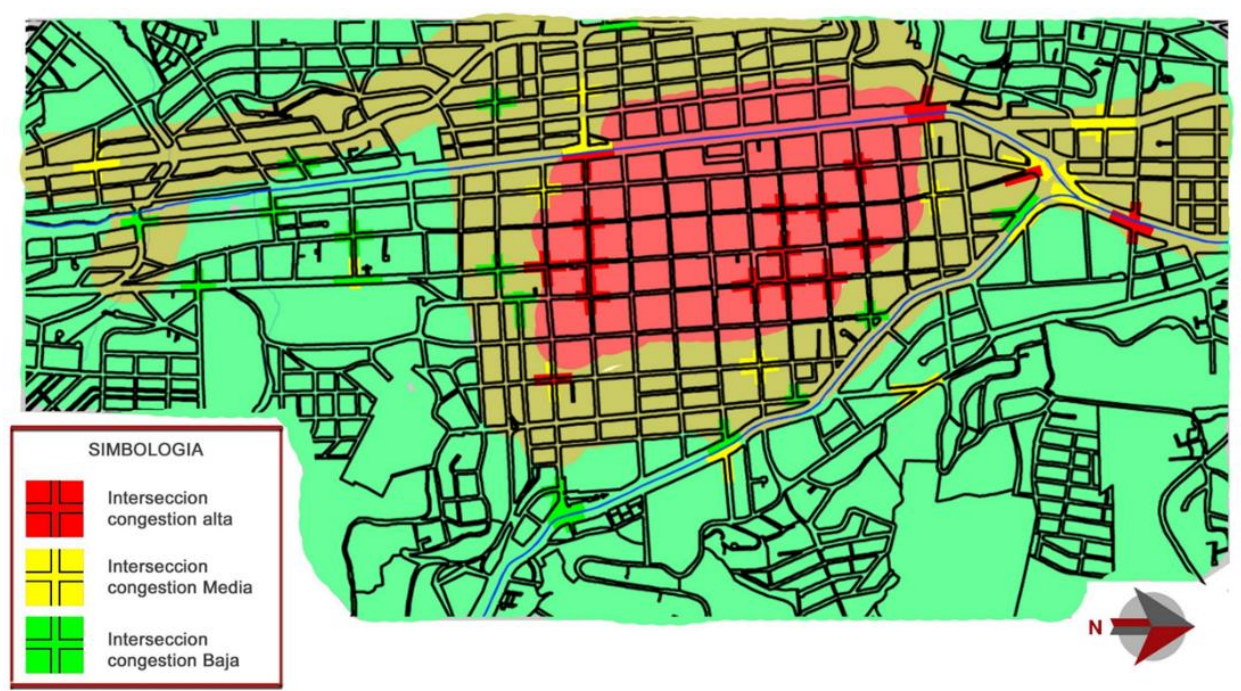

Ilustración 2: Niveles de congestión en el centro histórico de Loja generados por el software Synchro. Fuente: Software Synchro. Elabora por: El autor 
El análisis de los resultados e información obtenida en el desarrollo de la investigación, permitió generar una propuesta de implementación de ciclovías considerando cuatro instancias:

Primera instancia: Centro de la ciudad "Centro Histórico".- en esta instancia se propone complementar el proyecto del Municipio de Loja, implementando cinco circuitos más entre carriles bici (ilustración 3) y carriles compartidos (ilustración 4).
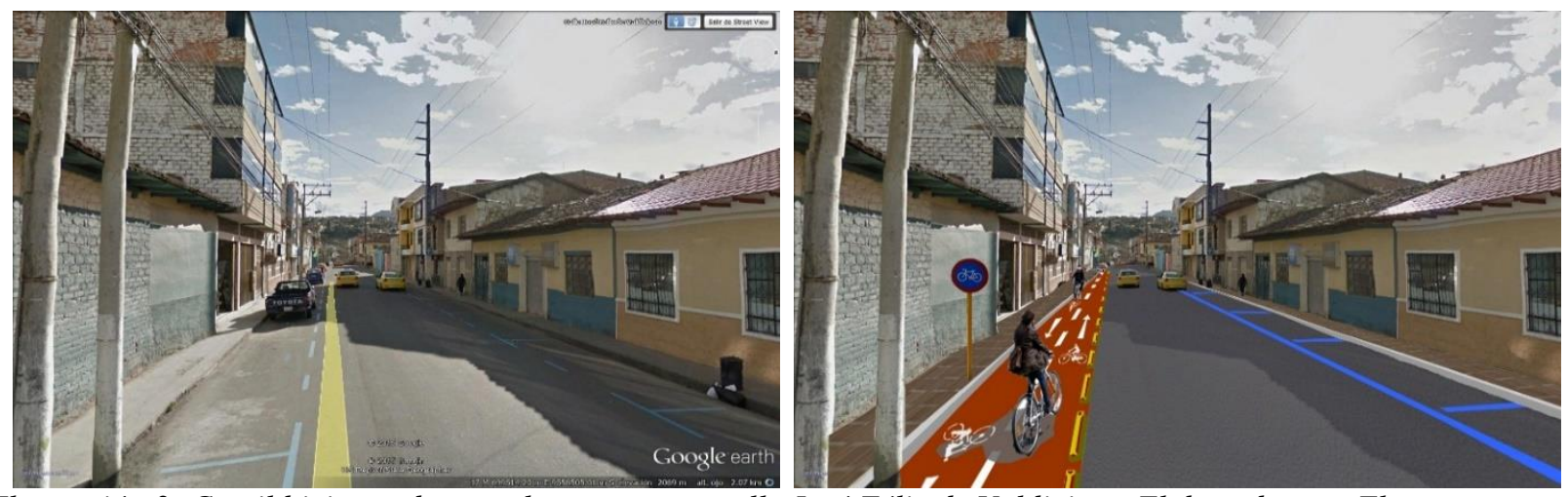

Ilustración 3: Carril bici, estado actual y propuesta, calle José Félix de Valdivieso. Elaborado por: El autor.

Segunda instancia: Barrios aledaños a los ejes vial troncal que atraviesa la ciudad de Norte a Sur y viceversa - "Relieves Mínimos".- Se propone cinco circuitos fundamentales, para la interconexión barrial y recreación tanto del oeste como del este de la ciudad (ilustración 5).
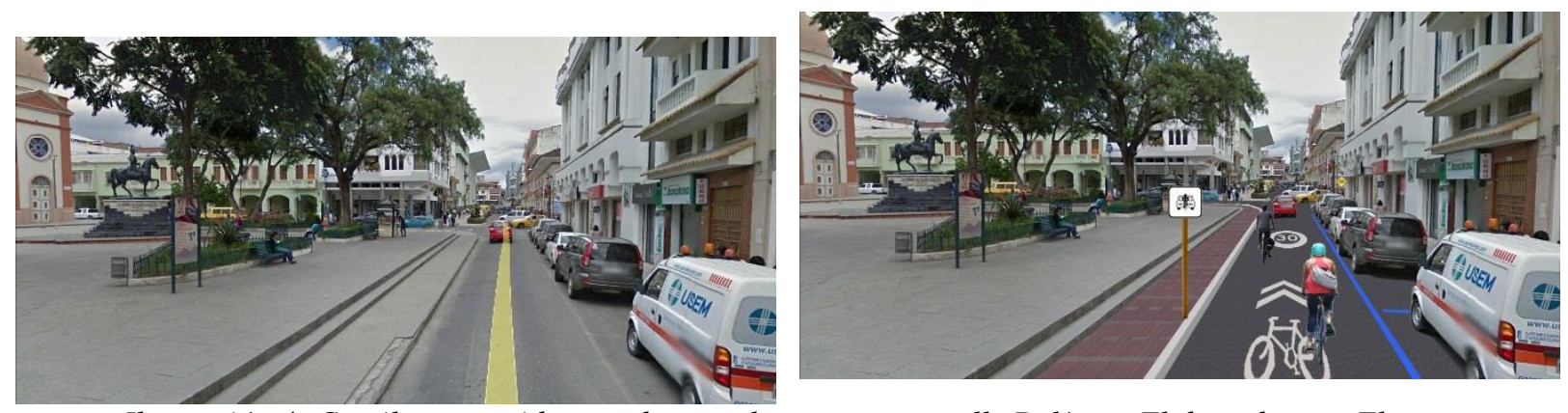

Ilustración 4: Carril compartido, estado actual y propuesta, calle Bolívar. Elaborado por: El autor.

Tercera y cuarta instancia: Barrios restantes de la ciudad cuyo acceso requiere atravesar vías que presentan "relieves pronunciados" y la interconexión vial.- En estas instancias se propone los circuitos ciclísticos necesarios que generen una red urbana de ciclovías que vinculen a todos los barrios de la ciudad. 

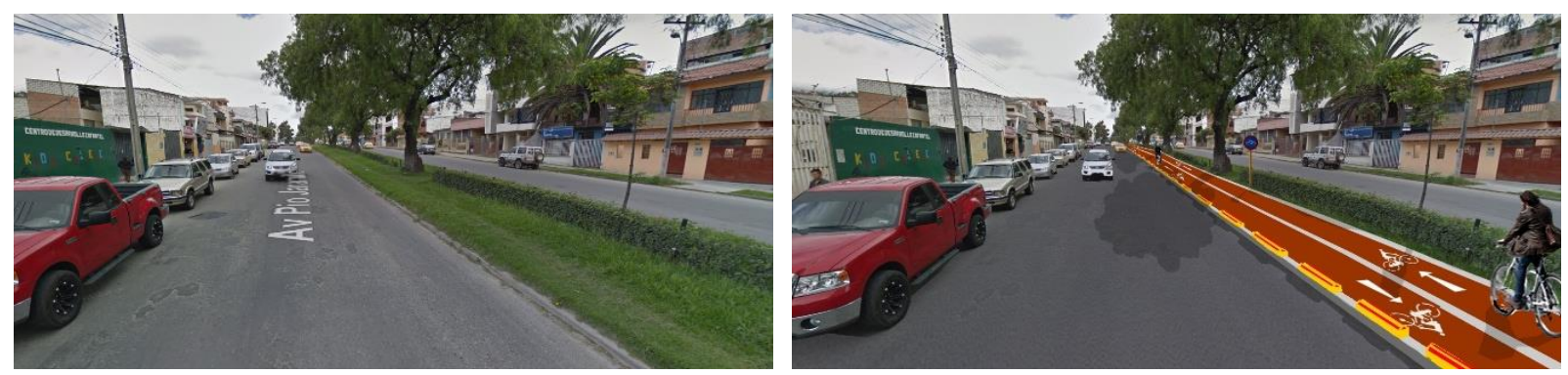

Ilustración 5: Carril bici, estad actual y propuesta Av. Pío Jaramillo Alvarado. Elaborado por: El autor.

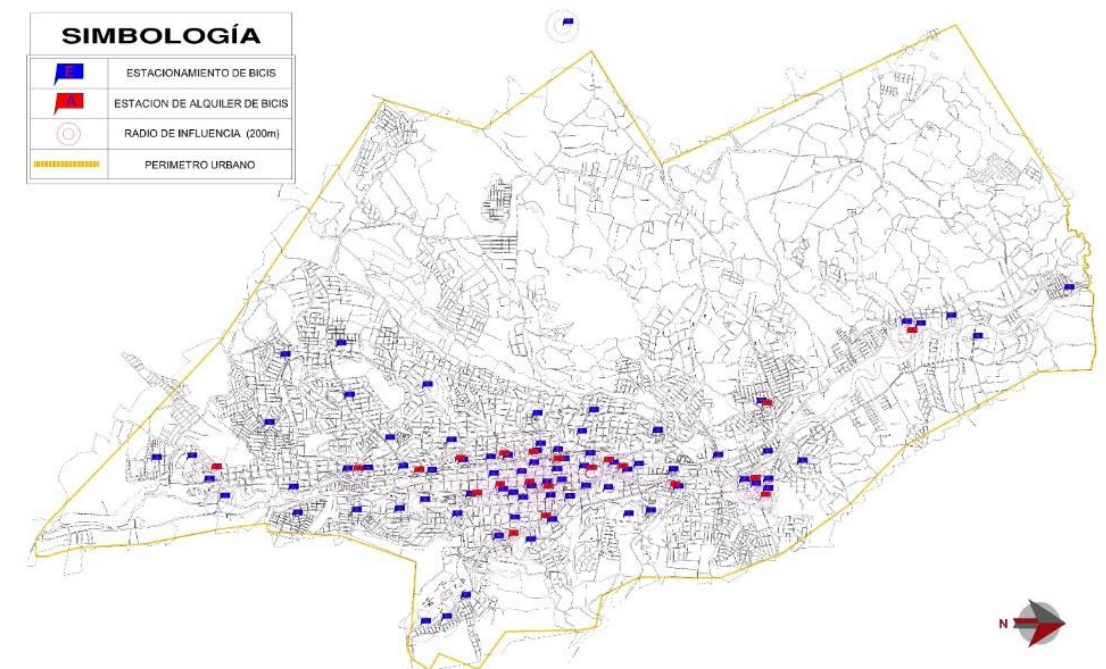

Ilustración 6: Propuesta de ubicación de estaciones de alquiler y estacionamientos de bicicleta. Elaborado por: El autor.

Las cuatro instancias propuestas se complementan con estacionamientos para bicicletas comunes y eléctricas, señalización vertical y horizontal, iluminación, estaciones de alquiler y stands de reparación (ilustración 6). La localización de estos servicios, fue producto del análisis de la ubicación de equipamientos urbanos y estaciones de autobús; con ello la cobertura del servicio ciclístico abarca todas las zonas de mayor densidad poblacional, y presta soluciones a las zonas de residencia que se encuentran en territorio de altas pendientes topográficas.

Los resultados demuestran la débil cultura vial que existe en los ciudadanos con respecto a la utilización de la bicicleta como modo de transporte, lo cual se ve reflejado en el poco respeto a los ciclistas y en la no existencia de infraestructura y mobiliario ciclístico en las calles de la ciudad, siendo necesario para contrarrestar esta realidad, promover el desarrollo sustentable de la ciudad de Loja, considerando por parte de las autoridades municipales la implementación de la presente propuesta, tomando en cuenta que la inversión se justifica de sobremanera, ya que el promover el uso de la bicicleta permitirá mejorar la salud de los habitantes, mejorar la dinámica urbana y el flujo vehicular y peatonal, disminuir la contaminación ambiental e incentivar el ahorro en las familias al no generarse gastos por transporte, consumo de combustible o tratamientos médicos de enfermedades producto del sedentarismo o la contaminación.

Es necesario acentuar que junto con la implementación del proyecto se debe implementar la norma legal correspondiente que regule la circulación vehicular, en la cual se considere e 
incentive el uso de bicicletas, se fortalezca la cultura vial y se genere las condiciones de seguridad y operatividad necesarias a favor de los ciclistas.

Es importante reconocer el uso de la bicicleta como un medio de transporte, y no solo como actividad recreativa; la manera de potenciar este hecho en ciudades como Loja, de características topográficas determinantes, es evaluar previamente la morfología urbana para clasificarla por su demanda y poder ofertar una correcta tipología; apuntando a una conectividad integral que abarca los viajes de origen y destino, especialmente en las ciudades centralizadas.

El presente trabajo de investigación permite reconocer la bondad de la aplicación del método de investigación científica para enfrentar problemas en las ciudades, como es el caso particular de la implementación de ciclovías en la ciudad de Loja, metodología de trabajo que puede ser replicada para buscar soluciones similares en otras ciudades, aportándose de manera global al desarrollo sustentable.

\section{Conclusiones}

De la población encuestada cuya edad oscila entre los 17 y 37 años de edad, el 87\% estaría dispuesta a utilizar infraestructura ciclística, si esta ofreciera espacios aptos con circuitos especiales, estacionamientos y seguridad.

La sectorización de la ciudad en cuatro instancias, es una alternativa favorable para plantear el diseño y planificación de la propuesta de una ciclovía que abarque toda la ciudad de Loja.

La contaminación ambiental es un factor fundamental dentro del planteamiento de este proyecto, ya que a través de la implementación de la ciclovía se lograría reducir en gran escala el daño al medio ambiente, producido por el exceso de tránsito vehicular, especialmente de taxis y vehículos privados

Se cumple la hipótesis de investigación, ya que la simulación de la propuesta utilizando el software Synchro, permite aseverar que la implementación de las ciclovias en las cuatro instancias propuestas, ayudará a reducir la congestión vehicular y el impacto ambiental, pudiendo considerarse la implementación del presente trabajo, como una de las mejores opciones para promover la movilidad sustentable utilizando la bicicleta en la ciudad de Loja.

La red ciclística propuesta en la ciudad de Loja, permitirá impulsar el turismo en la ciudad, tomando en cuenta que hay muchos circuitos que atraviesan sitios que son considerados turísticos en el sector.

\section{Bibliografía}

Acosta, G. (2004). Estrategias de transporte sustentable. Bogotá: Revista CIFRA.

Americas, C. R. (2012). Ciclovias Recreativas de las Americas. Ciclovias Recreativas de las Americas.org. 
Colmenares, G. (2007). Desarrollo sustentable y sostenible de sistemas de transporte público. Impacto en la gerencia, organización y liderazgo. Venezuela.

Gakenheimer, R. (1998). Los problemas de la movilidad en el mundo en desarrollo. EURE (Santiago), 24(72), 33-52.https://dx.doi.org/10.4067/S0250-71611998007200002

Hunt, E. (2005). La sustentabilidad y la ciudad: una aproximación a través de los sistemas complejos. Sustentabilidad. . San Francisco.

Ilustre Municipio de Loja. (2006). Centralidad urbana de organismos administrativos, gestión, servicio y comercio de Loja. Loja.

Ilustre Municipio de Loja. (2010). Sistema Municipal de Estacionamiento Rotativo Tarifario. Loja.

Instituto Ecuatoriano de Normalización. (2011). Reglamento Técnico Ecuatoriano para Señalización Vial. Ecuador.

Instituto Nacional de Estadisticas y Censos . (2010). Instituto Nacional de Estadisticas y Censos . Loja.

Naredo, J. (1997). La "ciudad sostenible": resumen y conclusiones. La construcción de la ciudad sostenible. . Espana: Trabalhos do Comité Habitat II.

Plan Nacional de Ciclovias del Ecuador, Ministerio de Transporte y Obras Publicas. (2012). Plan Nacional de Ciclovias del Ecuador. Guayaquil.

Programa de Naciones Unidas para el Medio Ambiente, Municipalidad de Loja y Naturaleza y Cultura Internacional. (2007). Perspectivas del Medio Ambiente Urbano: Geo Loja. Loja, Ecuador: PNUMA.

Romero, H., Toledo, X., \&Vasquez , A. (2001). Ecología urbana y gestión ambiental sustentable de las ciudades intermedias chilenas. Chile: Ambiente y Desarrollo.

Rueda, S. (2010). Un nuevo urbanismo para abordar los retos de la sociedad actual. El Urbanismo Ecológico. Conferencia ETSA. 